\title{
Uncommon and Atypical Sinonasal Masses: Diagnostic and Therapeutic Challenges
}

\author{
Rohit Sharma, Vinit Kumar Sharma, Rajneesh Madhok, Tanu Agarwal, Ashish Mehrotra, Anisha Kochhar
}

\begin{abstract}
Introduction: Sinonasal masses are a wide range of pathologies ranging from simple nasal polyps to rare tumors like esthesioneuroblastomas. Early symptoms of all of them are similar to common nasal and sinus problems, hence, producing a delay in diagnosis.
\end{abstract}

Materials and methods: All the patients presenting with sinonasal masses in the Department of ENT and Head/Neck Surgery, SRMS Institute of Medical Sciences, Bareilly, were included in the study. The study was carried out between January 2007 and December 2011. Detailed history and ENT examination were recorded. Radiological investigation was carried out in the form of computed tomographic (CT) scan of nose and paranasal sinuses. Final diagnosis was made by histopathological examination either before instituting any form of treatment or by a postsurgical biopsy where surgery was carried out. Immunohistochemistry was done in some cases as deemed necessary by the pathologist. Thus, their modes of presentation, radiological and histopathological profiles were studied along with dilemmas in diagnosis and management.

Results: A total of 189 nasal masses were observed during this period. Out of which 126 were diagnosed as nasal polyps and were excluded from the study. Thus, 63 uncommon nasal masses were seen in the study period. A large number of uncommon sinonasal masses had atypical presentation and/or presented in an advanced stage to pose a diagnostic and surgical challenge. Endoscopic techniques are becoming a gold standard in surgical resection in most of them. Interdepartmental cooperation is highly essential.

Keywords: Uncommon, Sinonasal mass, Histopathology, Computed tomographic scan, Endoscopic techniques.

How to cite this article: Sharma R, Sharma VK, Madhok R, Agarwal T, Mehrotra A, Kochhar A. Uncommon and Atypical Sinonasal Masses: Diagnostic and Therapeutic Challenges. Clin Rhinol An Int J 2012;5(3):114-117.

Source of support: Nil

Conflict of interest: None declared

\section{INTRODUCTION}

The sinonasal cavity extends from the nasal vestibule to the choana, opening posteriorly in the nasopharynx. The three turbinates, the inferior middle and the superior turbinate project from the lateral nasal wall with their corresponding meati. The pneumatized cavities surrounding the nose form the paranasal sinuses. The floor of the nasal cavity is the hard palate which also forms the roof of the oral cavity. The roof of the nasal cavity is formed by the base of the skull. Though with a complex anatomy sinonasal disease is one of the most common clinical head and neck problems. The majority of sinonasal pathology is inflammatory with neoplasms comprising approximately $3 \%$ of all head and neck tumors. Although sinus tumors are rare, they portend a poor prognosis, often due to advanced disease at diagnosis. ${ }^{1}$ The common symptoms are nasal obstruction, nasal discharge, epistaxis, headaches or facial pain, etc. Due to lack of awareness among primary health care providers, these patients are kept on being treated for common nasal conditions like rhinosinusitis having similar symptoms. A good clinical acumen, proper diagnostic facilities and a greater degree of surgical skill is required to manage these patients. However, they pose significant problems in management due their late presentation and juxtaposition to important anatomical structures, such as eye and brain. The increasing application of endonasal endoscopic techniques to their excision offers potentially similar scales of resection but with reduced morbidity. ${ }^{2}$

\section{MATERIALS AND METHODS}

This study was carried out in the Department of ENT and Head/Neck Surgery, SRMS Institute of Medical Sciences, Bareilly, Uttar Pradesh. All the patients in the study group were subjected to detailed history taking specifically asking for symptoms, such as nasal obstruction, nasal discharge, facial swelling/nasal deformity, facial pain or headache, epistaxis region, etc. as per the study form. Personal history including social and dietary habits was also noted. Complete ENT examination was documented and this was aided by nasal endoscopy wherever possible. After making a provisional diagnosis a contrast enhanced computed tomographic (CT) scan of nose and paranasal sinuses was obtained with coronal as well as axial sections. Some patients with these lesions were also subjected to magnetic resonance imaging (MRI). The final diagnosis was based on histopathological examination with few specimens subjected to immunohistochemistry as necessitated by the pathologist. Uncommon sinonasal masses included all the masses other than nasal polyps. Polyps arising from sphenoid sinus were an exception as they are rare and have atypical presentation. Also included in the study group were masses from other areas extending into nose or sinuses. A wide array of pathologies in sinonasal area was diagnosed. Thus, the primary objective of the study was to evaluate the incidence, clinical presentation, radiological and histopathological profiles of patients with uncommon and atypical sinonasal masses. The various factors causing delay 
in diagnosis and the challenges in management were also analyzed.

\section{RESULTS}

A total of 223 patients presented with a sinonasal mass or masses in the ENT outpatient department during the study period but only 189 patients had complete data to be recruited for the study. The youngest patient was a 4-yearold child and the eldest was 72 years of age. The most number of patients with sinonasal masses were seen in the 3rd decade of life followed by the 2nd decade (Table 1).

Of the 189 patients with sinonasal masses there were $57 \%$ males. There was a greater degree of male predominance in the uncommon and atypical sinonasal masses (Table 2). Around 70\% of the patients were from a low socioeconomic status.

Nasal obstruction was present in most of the patients (92\%). The other common nasal symptoms included nasal discharge (77\%), headache/facial pain (58\%) followed by hyposmia/anosmia (29\%). Eye symptoms were reported by $8.4 \%$ of the patients and most of them were malignant. Nasal/facial deformities were seen in significant number of patients in the study group. CNS symptoms were also reported. Surprisingly, only $76 \%$ of the patients were aware of development of a sinonasal mass (Table 3).

\section{Table 1: Age-related incidence of sinonasal masses}

\begin{tabular}{lcc} 
Age group & Number of patients & Percentage \\
\hline$<10$ & 3 & 1.5 \\
$11-20$ & 40 & 21 \\
$21-30$ & 36 & 19 \\
$31-40$ & 42 & 22 \\
$41-50$ & 35 & 18.5 \\
$51-60$ & 18 & 9.5 \\
$61-70$ & 12 & 6.3 \\
$71-80$ & 3 & 1.5 \\
\hline
\end{tabular}

Table 2: Gender distribution in sinonasal masses

\begin{tabular}{lrrr} 
Sinonasal masses & Males & Females & Total \\
\hline Overall & $108(57 \%)$ & $71(43 \%)$ & 189 \\
Nonmalignant & $31(70 \%)$ & $13(30 \%)$ & 44 \\
Malignant & $11(58 \%)$ & $8(42 \%)$ & 19 \\
\hline
\end{tabular}

Table 3: Distribution of symptoms in sinonasal masses

\begin{tabular}{lcc} 
Presenting symptoms & Number of patients & Percentage \\
\hline Nasal obstruction & 173 & 92 \\
Nasal discharge & 145 & 77 \\
Headache/facial pain & 87 & 46 \\
Anosmia/hyposmia & 55 & 29 \\
Epistaxis & 36 & 19 \\
Ear symptoms & 43 & 23 \\
Eye symptoms & 17 & 9 \\
CNS symptoms & 4 & 2 \\
Nasal/facial deformity & 25 & 13 \\
Awareness of sinonasal mass & 129 & 68 \\
\hline
\end{tabular}

The contrast enhanced CT scan was the radiological investigation of choice that was performed in all the patients with sinonasal masses. None of the patients with nasal polyps were subjected to MRI. However, $10 \%$ of the uncommon sinonasal masses underwent an MRI.

There was a greater degree of correlation between the clinical diagnosis and the histopathological diagnosis in the non-neoplastic masses. Whereas in the neoplastic sinonasal masses, a correct diagnosis was achieved on clinical basis in around $40 \%$ only. Insignificant number of nonmalignant sinonasal lesions required a repeat biopsy or an immunohistochemistry as compared to the malignant ones in which about $30 \%$ were subjected to immunohistochemistry.

In 63 patients (33\%), nasal masses were uncommon and atypical as per the study criteria and out of these $30 \%$ were malignant. Even the benign neoplastic sinonasal lesions had significant number of patients which were locally invasive, such as juvenile nasopharyngeal angiofibroma and to some extent the inverted nasal papilloma. The wide array of pathologies seen in sinonasal masses are depicted in Figures 1 and 2.

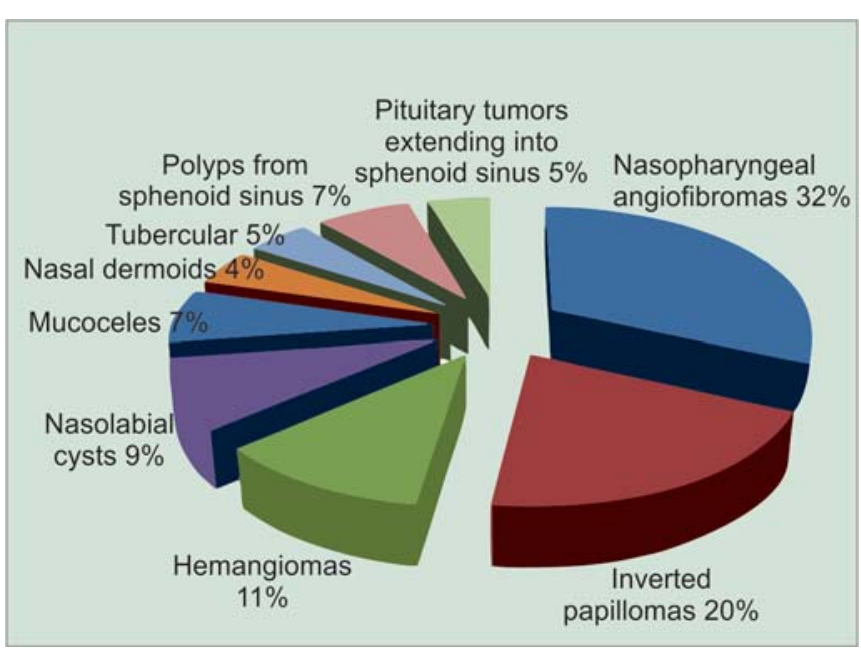

Fig. 1: Uncommon nonmalignant sinonasal masses

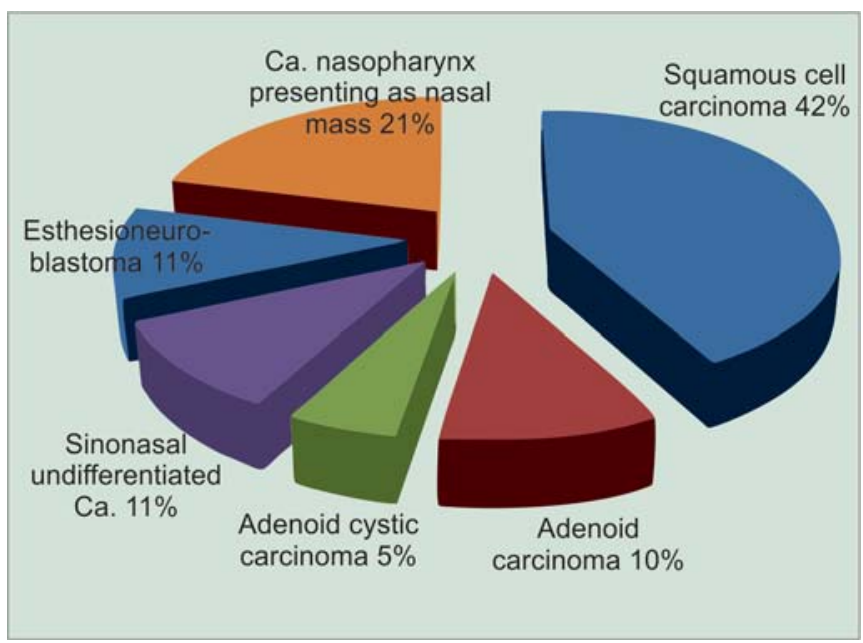

Fig. 2: Uncommon malignant sinonasal masses 
Surgery remained the treatment of choice and was performed in majority of patients with sinonasal masses. All except one uncommon nonmalignant sinonasal masses were operated as opposed to $47 \%$ of the malignant sinonasal masses. None of the malignancies of sinonasal areas, those operated, were given preoperative chemoradiation.

Endoscopic sinus surgery was performed in all patients with nasal polyps. Fifty-four percent of nonmalignant sinonasal masses were operated endoscopically. Endoscopic resection was not performed in any of the patients with malignant sinonasal masses.

Interdepartmental cooperation was required in significant number of uncommon nasal masses for the complete treatment. Highly skilled radiologists and pathologists with special inclination toward sinonasal pathologies are essential. Other departments involved were the neurosurgery for craniofacial resection in esthesioneuroblastoma (ENB) and also in pituitary tumors. Aid of plastic surgeon was required wherever, reconstruction was required. Prostheses were made by the dentists. Malignancies required chemoradiation (postoperative) during the treatment.

\section{DISCUSSION}

Nose and paranasal sinuses are involved by a plethora of conditions which arise primarily in this area or extend into from other surrounding areas. Males are more commonly afflicted by sinonasal masses. This has been documented by various other authors also. ${ }^{3,4}$ Male: Female ratio is more predominant in uncommon sinonasal masses as this group consists of malignancies and nasopharyngeal angiofibromas.

The early symptoms of a sinonasal mass are similar to those of simpler conditions, such as rhinosinusitis. By the time patient comes for examination to a specialist it is usually in an advanced stage. Only $76 \%$ of the patients in our study were aware of a nasal mass at the time of presentation and presumably the remaining $24 \%$ were being treated for common sinonasal diseases. Epistaxis, eye, ear and CNS symptoms were predominantly seen in uncommon sinonasal masses.

Nasal polyps are inflammatory outgrowths of paranasal sinus mucosa caused by chronic mucosal inflammation that typically arise from middle meatus and ethmoid region and appear as semitranslucent, pale gray growths in the nasal cavity. ${ }^{5}$ In most instances nasal polyps appear as a straightforward diagnosis. However, differential diagnosis is important to rule out congenital anomalies, as well as benign or malignant tumors. CT scan is helpful especially in determining the extent of disease and planning the surgical approach with regards to nasal polyps with endoscopic sinus surgery being the mainstay of surgical treatment. ${ }^{2,7}$ Endoscopic sinus surgery was performed in all patients having nasal polyps at our institute.

We evaluated all the patients with sinonasal masses with a CT scan with MRI aiding in some of them. Like most neoplasms, early detection improves prognosis, therefore clinicians and radiologists should be aware of features separating tumors from inflammatory sinus disease. ${ }^{2}$ This becomes especially confusing in patients presenting early where there is no bone erosion or extension into the surrounding structures. MRI was added as a diagnostic modality in the sinonasal masses showing doubtful intracranial or orbital invasions.

Seventy-three percent of the patients with sinonasal masses were found to be non-neoplastic. This was comparable to the studies done by various authors. ${ }^{6,7}$ The sinonasal malignancies reported with orbital invasion included the following histological groups, squamous cell carcinomas, neuroectodermal tumors like olfactory neuroblastomas, rhabdomyosarcomas, adenocarcinomas, etc. ${ }^{8,9}$ Similar correlation was found by us.

Most common malignancy of the sinonasal region is the squamous cell carcinoma. However, this complex anatomic region may represent the site of nonsquamous cell epithelial and nonepithelial malignant neoplasms of varying histogenesis, which are grouped under the term 'undifferentiated malignant neoplasms'. These undifferentiated malignancies share clinical and light microscopic features, which makes differentiation really difficult without the use of adjunct analyses, such as immunohistochemistry. ${ }^{10}$ Thus, $30 \%$ of the patients with sinonasal malignancies on histopathology had to be subjected to immunohistochemistry.

Certain studies show hemangioma as the most common benign neoplastic mass but it was nasopharyngeal angiofibroma in our as well in some other studies. ${ }^{6,11} \mathrm{~A}$ large number of our patients had extensive pathologies and were operated by external approaches but endoscopic removal was also done in a couple of patients.

Sinonasal inverted papillomas were primarily treated by endoscopic approach by us except for two patients where external approach like lateral rhinotomy was used. By the turn of the century endoscopic resection of sinonasal papillomas has become the standard treatment. ${ }^{12}$

There were two diagnosed cases of ENB on histopathology and immunohistochemistry. One of them underwent a craniofacial resection in collaboration with a neurosurgeon. The other was operated by a combined lateral rhinotomy and transpalatal approach. Both were given postoperative radiotherapy but are now lost in the long-term 
follow-up. ENB can be safely and effectively treated with craniofacial resection followed by irradiation. However, in experienced hands select cases of ENB can be safely excised and reconstructed endoscopically with comparable degrees of tissue removal as with external approaches. ${ }^{13,14}$ Six patients having more common malignancies of sinonasal area (squamous cell carcinoma, adenocarcinoma) were also operated by external approaches and the resection ranged from partial to total maxillectomies with or without orbital exenteration depending on the extent of the disease. Functional rehabilitation of total maxillectomy and orbital exenteration is essential to improve the quality of life and appearance of patients after surgery. ${ }^{8}$

\section{CONCLUSION}

A greater degree of suspicion and clinical acumen is required by otolaryngologists in patients with sinonasal masses, aided by dedicated radiologists and pathologists to confirm the diagnosis. The general practitioners should be made aware by the otolaryngology fraternity for obtaining a specialist opinion in patients with long standing nasal and sinus problems. An early diagnosis is of utmost importance to prevent greater morbidity and mortality. A greater degree of learning curve is required to surgically resect the uncommon sinonasal masses as it requires expertise in endoscopic, external, as well as microscopic techniques. In recent times endoscopic techniques have been applied even in lesions like extensive nasopharyngeal angiofibromas and ENBs. Institutions should have a dedicated team having representation from various departments for management of these uncommon and atypical sinonasal masses.

\section{REFERENCES}

1. Das S, Kirsch CFE. Imaging of lumps and bumps in the nose: A review of sinonasal tumours. Cancer Imaging 2005;5(1):167-77.

2. Lund VJ, Stammberger H, Nicolai P, et al. European position paper on endoscopic management of tumours of the nose, paranasal sinuses and skull base. Rhinol Suppl 2010 Jun 1;(22): 1-143.

3. Fasunla AJ, Lasisi AO. Sinonasal malignancies: A 10-year review in a tertiary health institution. J Natl Med Assoc 2007 Dec;99(12):1407-10.

4. Lund VJ. Diagnosis and treatment of nasal polyps. BMJ 1995;311:1411-14.

5. Georgy MS, Peters AT. Nasal polyps. Allergy Asthma Proc 2012 May-Jun;33 (Suppl 1):22-23.

6. Lathi A, Syed MMA, et al. Clinico-pathological profile of sinonasal masses: A study from a tertiary care hospital of India. Acta Otorhinolaryngol Ital 2011;31:372-77.
7. Bakari A, Afolabi OA, Adoga AA, et al. Clinico-pathological profile of sinonasal masses: An experience in national ear care center Kaduna, Nigeria. BMC Research Notes 2010;3:186.

8. Chu Y, Liu HG, Yu ZK. Patterns and incidence of sinonasal malignancy with orbital invasion. Chin Med J (Engl) 2012 May; 125(9):1638-42.

9. Habesoglu TE, Habesoglu M, Surmeli M, Uresin T, Egeli E. Unilateral sinonasal symptoms. J Craniofac Surg 2010 Nov;21(6):2019-22.

10. Wenig BM. Undifferentiated malignant neoplasms of the sinonasal tract. Archives Pathol Lab Med 2009 May;133(5):699-712.

11. Baradaranfar MH, Dabirmoghaddam P. Endoscopic endonasal surgery for resection of benign sinonasal tumors: Experience with 105 patients. Arch Iran Med 2006 Jul;9(3):244-49.

12. Woodworth BA, Bhargave GA, Palmer JN, et al. Clinical outcomes of endoscopic and endoscopic-assisted resection of inverted papillomas: A 15-year experience. Am J Rhinol 2007 Sep-Oct;21(5):591-600.

13. Nichols AC, Chan AW, Curry WT, et al. Esthesioneuroblastoma: The Massachusetts eye and ear infirmary and Massachusetts general hospital experience with craniofacial resection, proton beam radiation, and chemotherapy. Skull Base 2008 Sep;18(5):327-37.

14. Casiano RR, Numa WA, Falquez AM. Endoscopic resection of esthesioneuroblastomas. Am J Rhinol 2001 Jul-Aug;15(4): 271-79.

\section{ABOUT THE AUTHORS}

\section{Rohit Sharma (Corresponding Author)}

Associate Professor, Department of ENT and Head and Neck Surgery SRMS Institute of Medical Sciences, Ram Murti Puram, Bareilly243202, Uttar Pradesh, India, Phone: 9997449995, Fax: 05812582010 e-mail: rohitsharma.dr@gmail.com

\section{Vinit Kumar Sharma}

Assistant Professor, Department of ENT and Head and Neck Surgery SRMS Institute of Medical Sciences, Bareilly, Uttar Pradesh, India

\section{Rajneesh Madhok}

Associate Professor, Department of Radiology, SRMS Institute of Medical Sciences, Bareilly, Uttar Pradesh, India

\section{Tanu Agarwal}

Associate Professor, Department of Pathology, SRMS Institute of Medical Sciences, Bareilly, Uttar Pradesh, India

\section{Ashish Mehrotra}

Consultant, Department of ENT and Head and Neck Surgery, SRMS Institute of Medical Sciences, Bareilly, Uttar Pradesh, India

\section{Anisha Kochhar}

Resident, Department of ENT and Head and Neck Surgery, SRMS Institute of Medical Sciences, Bareilly, Uttar Pradesh, India 